DOI: 10.12957/demetra.2016.22340

\title{
À sombra do estereótipo de beleza: qualidade de vida e fatores associados em mulheres
}

\section{Under the shadow of the beauty stereotype: quality of life and associated factors in women}

\author{
Rayane Stephanie Gomes de Freitas ${ }^{1}$ \\ Renata Doratioto Albano ${ }^{2}$ \\ Diogo Thimoteo da Cunha ${ }^{3}$ \\ 1 Universidade Federal de São Paulo, Instituto de \\ Saúde e Sociedade. Santos-SP, Brasil. \\ ${ }^{2}$ Universidade Católica de Santos, Centro de \\ Ciências Aplicadas e Saúde. Santos-SP, Brasil. \\ ${ }^{3}$ Universidade Estadual de Campinas, Faculdade \\ de Ciências Aplicadas. Limeira-SP, Brasil. \\ Correspondência / Correspondence \\ Renata Doratioto Albano \\ E-mail: rdalbano@unisantos.br
}

\section{Resumo}

Objetivo: Neste estudo, avaliou-se a associação da composição corporal, aspectos gerais e socioeconômicos com a Qualidade de Vida de 308 mulheres adultas e 53 idosas atendidas em um Ambulatório de Nutrição. Métodos: A Qualidade Vida foi avaliada com o questionário WHOQOL-bref, da Organização Mundial da Saúde, composto pelos domínios: físico, psicológico, relações sociais e meio ambiente, e pelos quesitos autoavaliação e geral. Foram coletados dados de Índice de Massa Corporal, percentual de gordura e circunferência de cintura. Resultados: A obesidade e o sobrepeso foram observados em $47,4 \%$ das adultas e em $84,9 \%$ das idosas, respectivamente. Todas as mulheres apresentaram circunferência de cintura e percentual de gordura elevados. As idosas eutróficas $(p=0,03)$ e as adultas eutróficas $(p<0,001)$ possuíam maior Qualidade de Vida. Todos os domínios e quesitos da Qualidade de Vida mostraram correlação com o Índice de Massa Corporal, circunferência de cintura e percentual de gordura $(\mathrm{p}<0,001)$, isto é, quanto maiores estes valores, menor a Qualidade de Vida. A regressão múltipla expôs que o Índice de Massa Corporal $(\mathrm{p}<0,001)$, idade $(\mathrm{p}<0,001)$ e escolaridade $(\mathrm{p}<0,001)$ se relacionaram de forma independente com a Qualidade de Vida, sendo o Índice de Massa Corporal de maneira inversa e as duas últimas, de forma direta. Conclusão: Sugere-se que o aumento do Índice de Massa Corporal, por ser uma variável fisicamente perceptível às mulheres, repercuta negativamente sobre a Qualidade de Vida experienciada por esse grupo.

Palavras-chave: Mulheres. Qualidade de vida. Composição corporal. Imagem corporal. 


\section{Abstract}

Objectives: This study assessed the association among body composition, general and social aspects with Quality of Life of 308 adult and 53 elderly women treated in a nutrition outpatient cliniucc. Methods: Quality of Life was assessed using the WHOQOL-bref questionnaire of the World Health Organization, comprised of the physical, psychological, social relationships and environmental domains and by the self-evaluation and total categories. Data about Body Mass Index, body fat percentage and waist circumference were collected. Results: Obesity and overweight were present for the majority of adult (47.4\%) and elderly women $(84.9 \%)$, respectively. All women had their waist circumference and body fat percentage above the recommended values. Eutrophic elderly $(p=0.03)$ and eutrophic adults $(p<0.001)$ showed higher Quality of Life. All the domains and categories of Quality of Life showed a correlation with Body Mass Index, waist circumference and body fat percentage $(p<0.001)$. Thus, the higher these values, the lower the Quality of Life. The multiple regression model showed that Body Mass Index $(\mathrm{p}<0.001)$, age $(\mathrm{p}<0.001)$ and educational level $(\mathrm{p}<0.001)$ are related to Quality of Life in an independent manner, and Body Mass Index is inverse and the others in a direct way. Conclusion: Our study suggests that because the increase of Body Mass Index is a physically noticeable variable to women, it has negative impact on Quality of Life.

Key words: Women. Quality of life. Body composition. Body image.

\section{Introdução}

A Organização Mundial da Saúde (OMS) define "qualidade de vida” (QV) como:

Percepção individual da posição do indivíduo na vida, no contexto de sua cultura e sistema de valores nos quais ele está inserido e em relação aos seus objetivos, expectativas, padrões e preocupações. É um conceito de alcance abrangente afetado de forma complexa pela saúde física, estado psicológico, nível de independência, relações sociais e relações com as características do meio ambiente do indivíduo (World Health Organization, 1997). ${ }^{1}$

Embasados por essa definição, na década de 1990 foi criado pela Organização Mundial da Saúde um instrumento abreviado que busca, por meio de rápido preenchimento, avaliar o nível de qualidade de vida em quatro domínios (físico, psicológico, relações sociais e meio ambiente), denominado WHOQOL-BREF (World Health Organization Quality of Life - bref)., ${ }^{2,3}$ 
Para estudar qualidade de vida de mulheres é preciso abordar possíveis fatores inerentes a elas e que exerçam influência sobre suas vidas nos domínios físico, psicológico, relações sociais e meio ambiente. Alguns estudos buscaram elucidar algumas destas relações no que se reporta, por exemplo, às variáveis sociodemográficas e condições de saúde de idosas, ${ }^{4}$ insatisfação com o corpo ${ }^{5}$ e estilo de vida. ${ }^{6}$ A obesidade também se relaciona com uma baixa percepção de QV. ${ }^{7}$ Entretanto, acredita-se que mais que o estado nutricional, outros aspectos corporais podem se relacionar às percepções de QV em mulheres, principalmente aqueles mais associados à aparência, como gordura corporal e circunferência de cintura.

Nas últimas décadas no Brasil, as normas sociais quanto ao peso corporal aproximaram-se do ideal magro vigorante na Europa e América do Norte, o que ocasionou tanto um aumento do estigma acerca da obesidade quanto suscitou um aumento no impacto do Índice de Massa Corporal (IMC) sobre a percepção da qualidade de vida. ${ }^{7}$

A forma como os indivíduos se veem, ou seja, sua imagem corporal, envolve diversos fatores biopsicossociais, como a idade, prática de atividade física, número de doenças e percepção da saúde, sendo um dos mais importantes o estado nutricional. Assim, a percepção satisfatória da imagem corporal pode agir beneficamente ao estado de saúde dos indivíduos. ${ }^{8}$

Usualmente, as mulheres são mais preocupadas com o peso e forma corporal, no entanto, a busca constante por um "corpo perfeito", intensificada pela mídia em seus diversos meios, torna esse ideal quase inalcançável, o que resulta em insatisfação com a própria constituição física. ${ }^{9}$

Este artigo pretende falar da importância de outras facetas, ainda pouco exploradas, que se relacionam com os domínios da qualidade de vida e atuam para o bem-estar holístico dessa população. Ademais, destacamos o papel das medidas fisicamente perceptíveis como um fator de forte influência na concepção individual de saúde. Por meio de uma abordagem que detém uma visão plural a esta questão intenta-se contribuir com conhecimentos válidos para a real incorporação da noção de qualidade de vida quando aplicada à saúde da mulher.

Nesse tocante, este estudo busca avaliar a qualidade de vida e a sua associação com fatores socioeconômicos, de estilo de vida e composição corporal em um grupo populacional de mulheres adultas e idosas atendidas em um Ambulatório de Nutrição.

\section{Métodos}

Estudo transversal e exploratório, desenvolvido em um Ambulatório de Nutrição (AN) pertencente a uma Instituição de Ensino Superior (IES) particular, localizado na cidade de Santos-São Paulo/Brasil. O atendimento do AN é gratuito para os indivíduos de todas as faixas etárias, sejam eles sadios ou detentores de alguma doença. A demanda pelo AN ocorre tanto de 
modo voluntário como por orientação de profissionais da área da saúde. Grande parte do público atendido é morador do município de Santos/SP e das cidades circunvizinhas (São Vicente, Cubatão, Praia Grande e Guarujá).

A população de estudo foi constituída por todas as mulheres adultas (20 a 59 anos) e idosas ( $\geq 60$ anos) atendidas a partir de maio de 2012 até setembro de 2013. O ambulatório atende em média 300 indivíduos por ano e todas as mulheres (adultas e idosas) que foram atendidas no período foram convidadas a participar do estudo. A amostragem se deu por conveniência, com a exclusão de gestantes e lactantes.

Os dados socioeconômicos (grau de escolaridade, tipo de ocupação, renda familiar), composição corporal (peso, estatura, circunferência de cintura e percentual de gordura), gerais (motivo da procura pelo ambulatório de nutrição e presença de doença - relatada pelo próprio indivíduo) e de estilo de vida (prática de atividade física - tipo e frequência -, etilismo e tabagismo) foram coletados da anamnese nutricional específica para adultas e idosas, sendo estes referentes à primeira consulta nutricional (avaliação nutricional). Os dados sobre a qualidade de vida foram colhidos no momento da orientação nutricional, uma semana após a primeira consulta, uma vez que não ocorrem intervenções nutricionais nesse período.

O diagnóstico nutricional a partir do IMC (Índice de Massa Corporal - peso $(\mathrm{kg}) /$ altura $\left.^{2}(\mathrm{~m})\right)$ foi realizado de acordo com os pontos de corte estabelecidos pela World Health Organization ${ }^{10}$ para as adultas e segundo The Nutrition Screening Initiative (1994) para as idosas. ${ }^{11}$ Os procedimentos para a aferição do peso e da estatura seguiram os parâmetros estabelecidos pelo Ministério da Saúde pela Norma Técnica do Sistema de Vigilância Alimentar do Brasil. ${ }^{12}$ Para a aferição do peso corporal utilizou-se balança eletrônica Toledo®, com capacidade para $150 \mathrm{~kg}$ e precisão de $50 \mathrm{~g}$, e para a estatura, estadiômetro não portátil Tonelli®, com escala de 2,20 m.

A circunferência de cintura (CC) foi aferida por meio de uma fita métrica inelástica, e para a avaliação de risco para desenvolvimento de doenças cardiovasculares, empregou-se o ponto de corte recomendado pela World Health Organization. ${ }^{13}$ Os dados sobre o percentual de gordura foram obtidos por Impedância Bioelétrica (BIA), por meio do equipamento Biodynamics ${ }^{\circledR}$ modelo $310 \mathrm{e} /$ Body Composition Analyser. Para a realização da BIA, as participantes precisavam estar em jejum de alimentos e líquidos durante, no mínimo, quatro horas, não utilizarem marca-passo, não estarem gestantes ou durante o período menstrual. O percentual de gordura foi classificado de acordo com Jackson e Pollock (1978), citado por Heyward \& Stolarczyk. ${ }^{14}$ A Qualidade de Vida (QV) foi avaliada a partir de um questionário validado elaborado pela Organização Mundial da Saúde, denominado WHOQOL-BREF (abreviado), versão português, composto por 26 questões, sendo duas sobre o quesito autoavaliação e 24 divididas em quatro domínios (físico, psicológico, relações sociais e meio ambiente), com escore de 4 a 20 pontos em cada., ${ }^{2,15}$ Calculou-se também um quesito denominado geral, que consiste na média aritmética simples dos escores das 26 questões do instrumento. ${ }^{15}$ 
O domínio físico compreende facetas sobre dor e desconforto, energia e fadiga, sono e repouso, mobilidade, atividades cotidianas, dependência de medicamentos ou tratamentos e capacidade de trabalho; o domínio psicológico abrange as facetas sobre sentimentos positivos e negativos, autoestima, imagem corporal e aparência, pensar/aprender/memória/concentração e também sobre espiritualidade/religião/crenças pessoais. Facetas sobre as relações pessoais, suporte (apoio) social e atividade sexual fazem parte do domínio relações sociais e, por fim, no domínio meio ambiente encontram-se facetas sobre segurança física e proteção, ambiente no lar, recursos financeiros, cuidados de saúde e sociais, oportunidades de adquirir novas informações e habilidades, participação e oportunidades de lazer, transporte e ambiente físico (poluição/ruído/trânsito/clima). ${ }^{2}$

O cálculo dos escores e estatística descritiva de cada quesito e domínio do instrumento WHOQOL-BREF foi efetuado em planilha do aplicativo Excel®, versão 2007, com um instrumento elaborado por Pedroso et al. ${ }^{15}$

A análise estatística dos dados foi feita com o teste $t$-Student, para comparação de variáveis de grupos não relacionados (idosas x adultas; idosas eutróficas x idosas com sobrepeso), e a de variância (ANOVA), com um fator fixo e o teste de comparações múltiplas de Tukey, quando considerados dois ou mais grupos não relacionados (adultas eutróficas x com sobrepeso x obesas). Para medir a correlação entre as variáveis de composição corporal e a qualidade de vida, foi realizado o teste de correlação linear de Pearson. Foram elaborados modelos de regressão linear múltiplos para investigar a relação entre variáveis independentes e as variáveis de qualidade de vida (geral e domínios). Foram testadas como independentes nos modelos aquelas com coeficiente de correlação linear de Pearson maior que 0,20, e permaneceram apenas as que apresentaram o coeficiente de regressão estatisticamente significativo. A inserção de variáveis nos modelos se deu pelo método Stepwise: forward. A variável escolaridade entrou nos modelos com três categorias: ensino fundamental (completo ou incompleto), ensino médio (completo ou incompleto) e ensino superior (completo ou incompleto). A variável renda familiar foi inserida nos modelos também com três categorias: baixa ( $\leq 2$ salários mínimos), média ( $\geq 2$ a 4 salários mínimos) e alta ( $\geq 4$ salários mínimos). $\mathrm{O}$ ajuste dos modelos foi avaliado por análise de resíduos. Optou-se por não utilizar um modelo conceitual de regressão por se tratar de um estudo exploratório.

Os testes estatísticos foram realizados com auxílio do software SPSS - Statistical Package for Social Sciences®, versão 15.0., com nível de significância de 5\%.

A pesquisa foi aprovada pelo Comitê de Ética em Pesquisa da Instituição de Ensino Superior em 23/4/12 (protocolo: 27380). As participantes assinaram o Termo de Consentimento Livre e Esclarecido conforme resolução 196/96 do Conselho Nacional de Saúde (CNS) vigente no ano em questão. ${ }^{16}$ 


\section{Resultados e Discussão}

Participaram do estudo 308 adultas e 53 idosas, com médias (desvio padrão) de idade de 38,4 $(11,7)$ anos e $65,7(4,9)$ anos, respectivamente.

Para ambos os grupos etários, a maior parte recebia de 3 a 5 salários mínimos (42,9\%), não era etilista $(74,5 \%)$ e o principal motivo da procura pelo ambulatório foi o emagrecimento (50,9\%). A atividade física não era praticada por $60 \%$ das adultas e por $50,9 \%$ das idosas.

As adultas, em sua maioria, estavam cursando ou já tinham completado o ensino superior $(55,8 \%)$, enquanto a maior parte das idosas apresentou ensino médio completo (37,7\%). De forma geral, as mais instruídas (ensino médio e superior) tinham maior nível de QV geral $(\mathrm{p}<0,001)$, resultado encontrado também em outros estudos. ${ }^{17,18}$ Suscita-se a ideia de que quanto maior o nível educacional, maior o autocuidado com os diversos fatores que interferem na QV.

Em relação ao tabagismo, tanto adultas como idosas, em sua maioria, não possuíam este hábito $(94,4 \%)$ e as mulheres não tabagistas apresentaram-se com maior QV geral $(p=0,03)$. Os indivíduos, de maneira geral, detêm conhecimentos sobre os efeitos nocivos do tabagismo, e o fumante possui a noção do quão prejudicial são as substâncias presentes no cigarro, porém não quer ou não consegue parar com o vício, o que pode provocar um efeito negativo sobre a QV. De maneira semelhante, o domínio físico também é afetado, pois o tabagismo proporciona um risco maior para o aparecimento de doenças pulmonares, cardíacas, bronquite, dismenorreia, diminuição da fertilidade e antecipação da menopausa, ${ }^{19}$ isto é, há um decréscimo da percepção de saúde física na mulher fumante.

Em sua maioria, ambos os grupos etários apresentavam algum tipo de doença (59,8\%), exceto diabetes $(85,2 \%)$. A hipertensão arterial e a dislipidemia demonstraram-se ausentes para a maioria das adultas, com $61,8 \%$ e $80,6 \%$, respectivamente, e presentes para a maioria das idosas, com $66,7 \%$ e 54,9\%. Entre as mulheres portadoras e não portadoras de diabetes mellitus, hipertensão arterial e dislipidemia não houve diferenças significativas para a QV.

Quanto à presença de doenças crônicas não transmissíveis, as mulheres portadoras e não portadoras de diabetes mellitus, hipertensão arterial e dislipidemia não demonstraram diferenças significativas entre si para a QV (Tabela 1). Tal resultado já foi observado em outro estudo no Brasil. ${ }^{20}$ Levanta-se a hipótese de que este fato ocorra devido ao uso rotineiro de medicamentos para o controle dessas doenças não ser um complicador para as mulheres em estudo, fazendo com que não haja implicações negativas em sua QV. Levando-se em conta que as doenças crônicas não transmissíveis são consideradas silenciosas, pois os danos não são sentidos de forma marcante, principalmente se há o uso de medicamentos, até chegar a estágios mais avançados da doença, a diminuição da QV passa a ser imperceptível. 


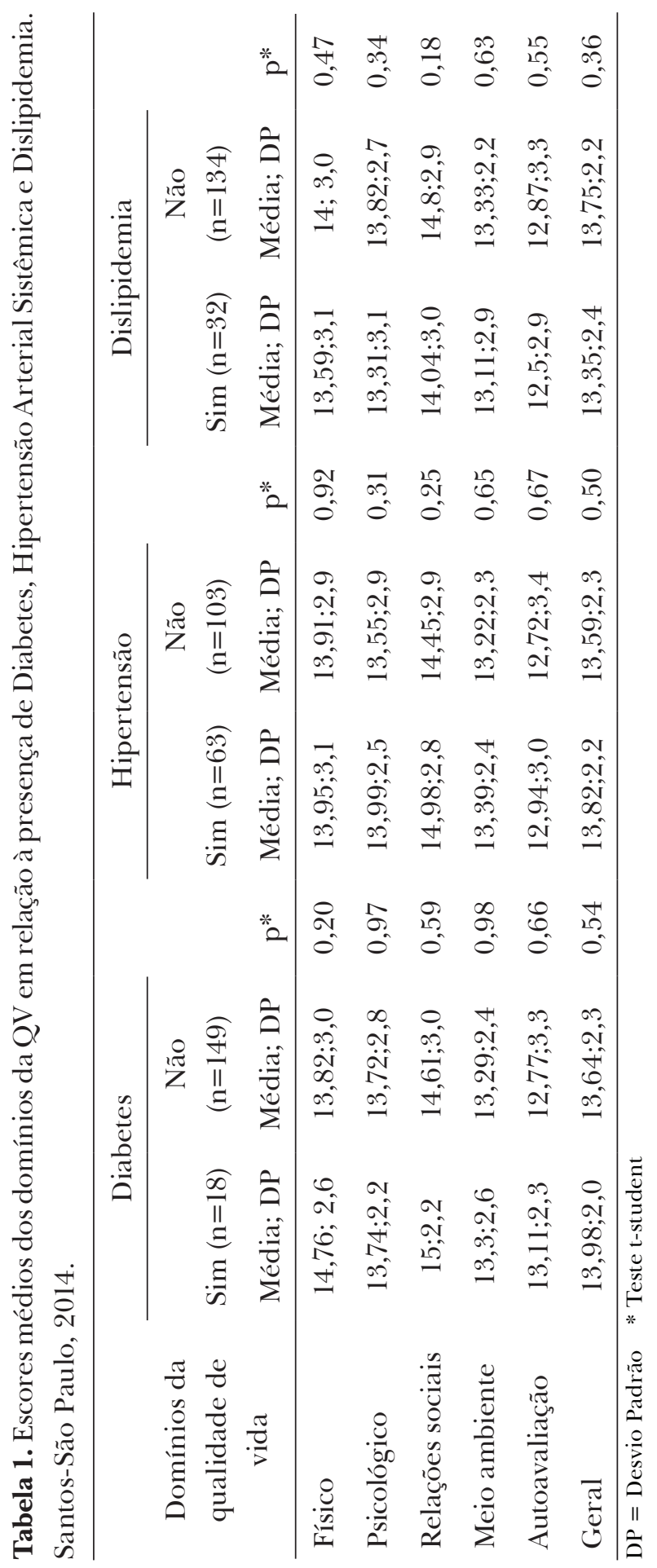


Em outro estudo brasileiro, o número de medicamentos utilizados por idosas não esteve significativamente associado aos domínios da qualidade de vida, excetuando-se o domínio físico, que pareceu melhorar para as que tomavam de um a dois medicamentos regularmente, o que sugere aos autores que haja atenuação dos desconfortos e dores provocados pela idade avançada. ${ }^{4}$ Levanta-se ainda a hipótese de que a utilização de remédios pode trazer conforto e segurança ao usuário, aumentando, consequentemente, suas percepções sobre a QV.

Acerca da qualidade de vida, as mulheres idosas demonstraram maior QV para o domínio meio ambiente $(\mathrm{p}=0,02)$ e para o quesito autoavaliação $(\mathrm{p}=0,03)$ quando comparadas às adultas, indicando que parecem estar mais engajadas na busca de uma melhor qualidade de vida, sendo integrantes de centros de convivência, praticando exercícios físicos ou outras ocupações sociais, que agora possuem maior disponibilidade de tempo para essas atividades. Menezes et al. ${ }^{8}$ observaram, em seu estudo de base populacional, que os idosos referiram satisfação com a sua imagem corporal, sugerindo que há uma maior aceitação deste aspecto frente ao envelhecimento, o que contribui significativamente para a autoestima e o autocuidado, repercutindo na busca por um melhor estado de saúde.

Ademais, as idosas possuem maior maturidade psicológica para aceitar os problemas advindos da idade e podem conviver com maior autoaceitação essa fase, uma vez que evidências sugerem que a maioria das pessoas se torna mais consciente, ${ }^{21,22}$ dominante, agradável e estável emocionalmente com o passar dos anos, ${ }^{21}$,bem como mais feliz, mais contente consigo mesma, centrada, descontraída e satisfeita com o que ela possui e menos preocupada com a produtividade em uma idade mais avançada. $^{23}$

Em relação ao estado nutricional, a obesidade (IMC $\geq 30 \mathrm{~kg} / \mathrm{m}^{2}$ ) apresentou-se em 47,4\% para as adultas e o sobrepeso (IMC $\geq 27 \mathrm{~kg} / \mathrm{m}^{2}$ ), para 84,9\% das idosas. Ambos os grupos etários demonstraram a CC muito elevada $(\geq 88 \mathrm{~cm})$, totalizando $73,9 \%$, assim como alto percentual de gordura (20-29 anos: $>31 \%$; 30-39 anos: $>32 \%$; $40-49$ anos: $>33 \%$; $50-59$ anos: $>34 \%$; $>60$ anos: $>35 \%$ ), em $76,2 \%$.

A classificação do IMC de adultas e idosas do presente estudo corrobora os dados encontrados na Pesquisa de Orçamentos Familiares (POF) 2008-2009, ${ }^{24}$ que mostrou que o excesso de peso em mulheres com 20 ou mais anos de idade aumentou de 28,7\%, em 1974-1975 para 48\%, em 2008-2009, e a obesidade, no mesmo período, cresceu mais de duas vezes, de $8 \%$ para 16,9\%. Os dados da Pesquisa Nacional de Saúde (PNS) 2013 também indicam diagnóstico similar, no qual a prevalência de excesso de peso para mulheres com idade igual ou superior a 20 anos foi de $59,8 \%$, e $25,2 \%$ de obesidade. ${ }^{25}$

As idosas eutróficas $(\mathrm{p}=0,03)$, assim como as adultas eutróficas $(\mathrm{p}<0,001)$, apresentaram maior QV (Tabelas 2 e 3). No que se refere à composição corporal, para as adultas foi verificado que quanto maior a classificação do IMC, menor a QV, resultado também encontrado em outro estudo. ${ }^{7}$ Quanto ao resultado do domínio psicológico e quesitos autoavaliação e geral, observou-se que quanto maior 
a classificação do IMC, menor a QV. Pode-se sugerir que quanto maior o excesso de peso, mais desmotivada emocionalmente a mulher se torna, pois ela não possui o estereótipo considerado agradável e aceitável perante a sociedade, enquanto a mulher eutrófica apresenta-se com maior QV e bem-estar em todos os aspectos e consegue se reconhecer como uma pessoa saudável.

Tabela 2. Escores médios dos domínios da QV em relação ao estado nutricional das idosas. Santos-São Paulo, 2014.

\begin{tabular}{lccccccc}
\hline & \multicolumn{5}{c}{ Eutrófico } & \multicolumn{5}{c}{ Sobrepeso } \\
Domínio & $\mathrm{n}$ & Média & $\mathrm{DP}$ & $\mathrm{n}$ & Média & $\mathrm{DP}$ & $\mathrm{p}^{*}$ \\
Físico & 8 & 16,94 & 2,07 & 45 & 13,61 & 2,73 & $<\mathbf{0 , 0 1}$ \\
Psicológico & 8 & 15,67 & 1,71 & 44 & 14,05 & 2,69 & 0,1 \\
Relações sociais & 8 & 15,17 & 2,75 & 44 & 14,74 & 2,71 & 0,68 \\
Meio ambiente & 8 & 14,75 & 2,30 & 45 & 13,99 & 2,41 & 0,41 \\
Autoavaliação & 8 & 16,5 & 1,77 & 44 & 13,64 & 2,77 & $<\mathbf{0 , 0 1}$ \\
Geral & 8 & 15,72 & 1,52 & 45 & 13,97 & 2,17 & $\mathbf{0 , 0 3}$ \\
\hline
\end{tabular}

DP $=$ Desvio Padrão $*$ Teste t-student

Valores de p em negrito representam diferença estatisticamente significativa.

Tabela 3. Escores médios dos domínios da QV em relação ao estado nutricional das adultas. Santos-São Paulo, 2014.

\begin{tabular}{|c|c|c|c|c|c|c|c|c|c|c|c|c|c|}
\hline \multirow[b]{2}{*}{ Domínios } & \multicolumn{4}{|c|}{ Eutrófico } & \multicolumn{4}{|c|}{ Sobrepeso } & \multicolumn{4}{|c|}{ Obesidade } & \multirow[b]{2}{*}{$\mathrm{p}^{*}$} \\
\hline & $\mathrm{n}$ & Média & DP & & $\mathrm{n}$ & Média & DP & & $\mathrm{n}$ & Média & DP & & \\
\hline Físico & 53 & 15,67 & 2,38 & $\mathrm{a}$ & 109 & 14,38 & 2,62 & $\mathrm{~b}$ & 145 & 13,67 & 2,97 & $\mathrm{~b}$ & $<0,001$ \\
\hline Psicológico & 52 & 15,27 & 1,88 & $\mathrm{a}$ & 106 & 14,08 & 2,21 & $\mathrm{~b}$ & 146 & 13,17 & 2,68 & c & $<0,001$ \\
\hline Relações sociais & 53 & 15,50 & 2,41 & a & 109 & 15,08 & 2,81 & $\mathrm{a}$ & 146 & 14,52 & 2,92 & $\mathrm{a}$ & 0,063 \\
\hline Meio Ambiente & 53 & 14,16 & 1,97 & $\mathrm{a}$ & 109 & 13,21 & 2,38 & $\mathrm{~b}$ & 146 & 13,07 & 2,29 & $\mathrm{~b}$ & 0,01 \\
\hline Auto-avaliação & 53 & 15,36 & 2,18 & $\mathrm{a}$ & 109 & 13,54 & 2,78 & $\mathrm{~b}$ & 146 & 11,97 & 3,14 & c & $<0,001$ \\
\hline Geral & 53 & 15,07 & 1,54 & $\mathrm{a}$ & 109 & 13,97 & 1,96 & $\mathrm{~b}$ & 146 & 13,33 & 2,13 & c & $<0,001$ \\
\hline
\end{tabular}

$\mathrm{DP}=$ Desvio Padrão

*Análise de variância com um fator fixo e teste de Tukey.

Letras diferentes na mesma linha indicam diferenças significativas $(p<0,01)$.

Valores de $\mathrm{p}$ em negrito representam diferença estatisticamente significativa. 
Para além dos importantes aspectos deletérios em relação à saúde, sabe-se que o excesso de peso provoca mudanças psicológicas e sociais em mulheres. Jackson \& Beeken et al. ${ }^{26}$ observaram que a obesidade, a discriminação de peso e o bem-estar psicológico estão inter-relacionados, apontando efeitos indiretos da obesidade, por meio da percepção da discriminação de peso, na qualidade de vida, na satisfação com a vida e em sintomas depressivos. Ainda, este referido estudo destacou o papel da discriminação de peso percebida, explicando cerca de $40 \%$ da associação da obesidade com o bem-estar psicológico. ${ }^{26}$

O estigma do peso, além de intensificar comportamentos não saudáveis, gera implicações negativas para a saúde pública, uma vez que ameaça a saúde física e psicológica de indivíduos obesos, cria obstáculos à implementação de medidas efetivas para a prevenção da doença e exaspera as disparidades em saúde. ${ }^{27}$

Acerca disso, Agrawal et al. ${ }^{28}$ estudaram fatores psicossociais associados a mulheres obesas em um país em desenvolvimento e verificaram que problemas cotidianos (caminhar, subir escadas, agachar e realizar atividades domésticas), insatisfação com a imagem corporal, insatisfação sexual, estigma e discriminação estavam relacionados com IMC aumentado. Além disso, as mulheres pesquisadas relataram sofrer com gozações e comentários depreciativos. ${ }^{28}$

Todos os domínios e quesitos da QV demonstraram correlação negativa com o IMC, CC e percentual de gordura ( $\mathrm{p}<0,001)$, isto é, quanto maiores estes valores, menor a QV (Tabela 4). O IMC apresentou-se como a variável significativa no modelo de regressão, o que pode indicar que medidas fisicamente perceptíveis às mulheres influenciam muito mais em seu julgamento da QV em todos os domínios que aquelas que não são visivelmente aparentes, uma vez que podem se sentir constrangidas e incomodadas por não contemplarem o estereótipo de beleza vigorante. 
Tabela 4. Correlação entre os domínios e quesitos da QV com o IMC, circunferência de cintura e percentual de gordura das adultas e idosas. Santos-São Paulo, 2014.

\begin{tabular}{lcccc}
\hline \multicolumn{1}{c}{ Domínio } & IMC & $\begin{array}{c}\text { Circunferência } \\
\text { de Cintura }\end{array}$ & $\begin{array}{c}\text { Percentual de } \\
\text { Gordura }\end{array}$ & $\mathrm{p}^{*}$ \\
\hline Físico & $\mathrm{r}$ & $\mathrm{r}$ & $\mathrm{r}$ & \\
Psicológico & $-0,35$ & $-0,33$ & $-0,27$ & $<0,001$ \\
Relações Sociais & $-0,32$ & $-0,30$ & $-0,22$ & $<0,001$ \\
Meio ambiente & $-0,20$ & $-0,22$ & $-0,15$ & $<\mathbf{0 , 0 0 1}$ \\
Autoavaliação & $-0,20$ & $-0,18$ & $-0,12$ & $<\mathbf{0 , 0 0 1}$ \\
Geral & $-0,42$ & $-0,42$ & $-0,30$ & $<\mathbf{0 , 0 0 1}$ \\
\hline
\end{tabular}

* Correlação de Pearson

Valores de p em negrito representam diferença estatisticamente significativa.

A mulher sente com maior profundidade a sobrecarga de uma beleza estereotipada imposta pela mídia em seu cotidiano e a pressão para obter um corpo perfeito. ${ }^{9}$ Contudo, quando falha na busca ou manutenção do estado nutricional adequado, sente-se frustrada, o que pode atingir diretamente sua QV. ${ }^{8}$

Cohen et al., ${ }^{29} \mathrm{em}$ seu estudo, sugerem que sites de rede social são fatores-chave que precisam ser considerados no desenvolvimento da insatisfação da imagem corporal. Ainda, pontuam que a exposição ao Facebook é, no mínimo, igual à exposição à mídia convencional quanto aos efeitos prejudiciais sobre a insatisfação da imagem corporal via comparação de aparência.

Ao passo que a imagem corporal mostra-se como mediadora para o bem-estar psicológico e para a habilidade de um indivíduo em manter a perda de peso, ${ }^{30}$ alguns estudos evidenciam que a insatisfação com essa imagem também está associada com acentuado prejuízo em vários aspectos da qualidade de vida de mulheres. Jackson \& Janssen et al. ${ }^{31}$ apontam que baixos níveis de atratividade percebida e imagem corporal estão correlacionados a sintomas depressivos, e Mond et al. ${ }^{5}$ enfatizam a insatisfação com a imagem corporal no que concerne aos itens relacionados à saúde mental, funcionamento psicossocial e, no mínimo, com alguns aspectos da saúde física, independente da sua associação com o peso corporal.

Não obstante, outra faceta explorada por Runfola et al. ${ }^{32}$ ressalta que as mulheres parecem exercer considerável esforço, contradizendo o estereótipo de resultado fácil, para manter um nível favorável de satisfação e, ocasionalmente, experimentam insatisfação com outros aspectos de sua aparência, especialmente aqueles afetados pelo envelhecimento. 
Estudos recentes evidenciam associações entre a insatisfação corporal e comportamentos não saudáveis relacionados ao peso, em especial, o maior uso de dietas ${ }^{32,33}$ e comportamentos danosos para controle de peso. ${ }^{32}$ Blake et al. ${ }^{33}$ observaram que aqueles que estavam insatisfeitos com o seu peso tenderam a realizar pequenos lanches em maior quantidade e, inversamente, consumir menos refeições.

Foram elaborados modelos considerando três variáveis dependentes: qualidade de vida geral, domínio físico e domínio psicológico. Os modelos das variáveis: domínio meio ambiente e domínio relações sociais não apresentaram ajuste adequado. O primeiro modelo de regressão múltiplo verificou que as variáveis IMC $(\mathrm{p}<0,001)$, idade $(\mathrm{p}<0,001)$ e escolaridade $(\mathrm{p}<0,001)$ se relacionam de forma independente com a QV geral, sendo o IMC de maneira inversa e as duas últimas, de forma positiva. Para o modelo domínio físico, foi verificada a relação inversa do IMC ( $\mathrm{p}<0,001)$ e positiva renda familiar $(\mathrm{p}=0,02)$. No terceiro modelo com o domínio psicológico como variável dependente, foi verificada a relação inversa com o IMC $(<0,001)$ e positiva com a idade $(\mathrm{p}<0,01)$ (Tabela 5$)$.

Tabela 5. Modelo de regressão linear múltiplo entre a variável qualidade de vida geral e as variáveis circunferência de cintura, idade, escolaridade e renda familiar das adultas e idosas. Santos-São Paulo, 2014.

\begin{tabular}{|c|c|c|c|}
\hline Modelo & Variáveis & $\begin{array}{c}\text { Coeficientes } \\
\text { padronizados }(\beta)\end{array}$ & $\mathrm{p}^{*}$ \\
\hline \multirow{5}{*}{$\begin{array}{l}\text { Modelo } 1 \text { - } \\
\text { Qualidade de } \\
\text { vida geral }\end{array}$} & Constante & 14,32 & $<0,01$ \\
\hline & IMC $\left(\mathrm{kg} / \mathrm{m}^{2}\right)$ & $-0,33$ & $<0,01$ \\
\hline & Idade (anos) & 0,20 & $<0,01$ \\
\hline & Escolaridade (três categorias) & 0,17 & $<0,01$ \\
\hline & Renda familiar (três categorias)** & 0,03 & 0,47 \\
\hline \multirow{3}{*}{$\begin{array}{l}\text { Modelo } 2 \text { - } \\
\text { Domínio físico }\end{array}$} & Constante & 17,54 & $<0,001$ \\
\hline & IMC $\left(\mathrm{kg} / \mathrm{m}^{2}\right)$ & $-0,31$ & $<0,001$ \\
\hline & Renda familiar (três categorias) & 0,12 & 0,02 \\
\hline \multirow{3}{*}{$\begin{array}{l}\text { Modelo } 3 \\
\text { - Domínio } \\
\text { psicológico }\end{array}$} & Constante & 16,69 & $<0,001$ \\
\hline & IMC & $-0,34$ & $<0,001$ \\
\hline & Idade (anos) & 0,15 & 0,004 \\
\hline
\end{tabular}

\footnotetext{
*Modelo de regressão linear múltiplo

**Variável de ajuste

Valores de p em negrito representam diferença estatisticamente significativa.
} 
A respeito da relevância das variáveis escolaridade e renda, Prättälä et al., ${ }^{34} \mathrm{em}$ seu estudo, enfatizam que recursos culturais associados a uma maior escolaridade e os recursos materiais a uma maior renda parecem ser elementos-chave para a manutenção do peso corpóreo normal. Ainda, ressaltam que o baixo nível socioeconômico, para além dos efeitos sobre comportamentos saudáveis e peso corpóreo, diminui também o controle pessoal dos indivíduos sobre sua vida e trabalho. ${ }^{34}$

Dentro dessa ótica, Mansfield et al., ${ }^{35}$ que estudaram um grupo de mães, observaram que o estigma social de comprovar a baixa renda foi um fator impeditivo para que algumas mulheres buscassem facilitadores governamentais para a prática de atividade física. Ademais, destacaram outras barreiras, como o alto custo com cuidados para crianças, principalmente para as que tinham mais de um filho, assim como os custos com o transporte até o local e com as roupas para a prática em questão. ${ }^{35}$

No que se refere à idade e qualidade de vida, resultados contrastantes foram encontrados por Murtagh et al., ${ }^{36}$ que avaliaram idosos, em sua maioria mulheres, e notaram que essas estavam 2,2 vezes mais propensas à inatividade que os homens e que características como viver sozinha, ser aposentada e autoavaliar a saúde emocional como baixa estavam associadas com a inatividade física, e que esta declina com o passar dos anos. Não obstante, Bryla et al., ${ }^{37}$ que igualmente pesquisaram idosos, na sua maioria mulheres, enfatizam que a qualidade de vida desse grupo depende de vários fatores, como a educação, renda e relacionamentos familiares, porém o destaque principal deve ser conferido aos aspectos ligados à saúde física e mental. Tais dados corroboram os resultados encontrados no presente estudo, ressaltando a importância da repercussão dos domínios físico e psicológico na manutenção da saúde e qualidade de vida.

Convém realçar o importante papel do IMC como variável independente quando relacionada à qualidade de vida geral das mulheres. Acerca disso, destaca-se que mulheres satisfeitas com seu tamanho corporal reportam menos preocupação com o peso e forma do corpo, ${ }^{32}$ bem como quando satisfeitas com o peso, tendem a praticar mais atividade física e a ter mais saúde. ${ }^{33}$ Ainda, Mintem et al. ${ }^{9}$ notaram que as mulheres cujo IMC aumentou ao longo do tempo tornaram-se mais insatisfeitas com seu tamanho corporal e, consequentemente, com sua imagem corporal.

Intervenções para a perda de peso podem melhorar a imagem corporal de adultos com sobrepeso ou obesidade. ${ }^{30}$ Para mulheres com excesso de peso, a percepção de peso superior ao ideal pode conduzi-las a comportamentos saudáveis, ao passo que para mulheres eutróficas a mesma situação merece mais atenção, uma vez que esse grupo está mais predisposto a comportamentos de risco. ${ }^{9}$

O filósofo e sociólogo Pierre Bourdieu, ${ }^{38} \mathrm{em}$ sua teoria social, coloca que a experiência feminina do corpo consolida-se em uma experiência do corpo-para-o-outro, isto é, por serem constantemente expostas à objetivação, tornam-se um objeto simbólico, o que as coloca em permanente estado de insegurança corporal. Ainda, destaca que a probabilidade de vivenciar o descontentamento 
do próprio corpo intensifica-se quanto maior a distância entre o corpo socialmente exigido e o feedback do próprio corpo dado por olhares e julgamentos de outrem.

Portanto, sugere-se que a alta intensidade da relação das medidas fisicamente perceptíveis com a qualidade de vida permeia os campos da apreciação e veredito sociais, o que eleva a importância da imagem corporal e aparência na concepção individual de saúde, ecoando fortemente na qualidade de vida dessa população.

\section{Conclusões}

Todos os domínios e quesitos da qualidade de vida apresentaram correlação negativa com IMC, circunferência de cintura e percentual de gordura. As variáveis IMC, idade e escolaridade mostraram-se associadas de maneira independente à qualidade de vida geral das mulheres, sendo a primeira de maneira negativa e as duas últimas, de forma positiva. Sugere-se que o aumento do IMC, por ser uma variável fisicamente perceptível às mulheres, repercuta negativamente sobre a qualidade de vida.

Com um maior conhecimento sobre os fatores que interferem na saúde da população feminina adulta e idosa, os profissionais de saúde poderão amplificar e melhorar a sua atuação para abarcar, o quão possível, as diversas dimensões que influenciam na QV deste gênero, com ênfase no estado nutricional e imagem corporal, a fim de planejar tratamentos e ações para prevenção de doenças mais adequados a essa população.

\section{Referências}

1. World Heatlh Organization. Programme on Mental Health. WHOQOL measuring quality of life [Internet]. Geneva: WHO; 1997 Disponível em: http://www.who.int/mental_health/media/68.pdf

2. Fleck MPA, Louzada S, Xavier M, Chachamovich E, Santos L, Pinzon V. Aplicação da versão em português do instrumento abreviado de avaliação da qualidade de vida "WHOQOL-bref." Rev Saude Publica 2000; 34(2):178-83.

3. World Health Organization. WHOQOL Group. Development of the World Health Organization WHOQOL-bref quality of life assessment. Psychol Med 1998; 28: 551-58.

4. Vagetti GC, Barbosa Filho VC, Moreira NB, De Oliveira V, Mazzardo O, De Campos W. Condições de saúde e variáveis sociodemográficas associadas à qualidade de vida em idosas de um programa de atividade física de Curitiba, Paraná, Sul do Brasil. Cad Saude Publica 2013; 29(5):955-69.

5. Mond J, Mitchison D, Latner J, Hay P, Owen C, Rodgers B. Quality of life impairment associated with body dissatisfaction in a general population sample of women. BMC Public Health BMC Public Health 2013; 13:920. 
6. Salehi A, Harris N, Sebar B, Coyne E. Self-perception of quality of life and its association with lifestyle behaviours of young Iranian women. Iran J Public Health 2015; 44(3):332-40.

7. Pimenta FBC, Bertrand E, Mograbi DC, Shinohara H, Landeira-Fernandez J. The relationship between obesity and quality of life in Brazilian adults. Front Psychol. 2015; 6:966.

8. Menezes TN, Brito KQD, Oliveira ECT, Pedraza DF. Percepção da imagem corporal e fatores associados em idosos residentes em município do nordeste brasileiro: um estudo populacional. Cienc Saude Coletiva 2014; 19(8):3451-60.

9. Mintem GC, Gigante DP, Horta BL. Change in body weight and body image in young adults: a longitudinal study. BMC Public Health 2015; 15:222.

10. World Health Organization. Physical status: the use and interpretation of anthropometry. Geneva: WHO; 1995. 452 p. WHO Technical Report Series, n. 854.

11. The Nutrition Screening Initiative. The American Dietetic Association. National Council on Aging Inc. Incorporating Nutrition Screening and Interventions into Medical Practice A Monograph for Physicians. Washington D.C.: American Academy of Family Physicians; 1994.

12. Brasil. Ministério da Saúde. Orientações para a coleta e análise de dados antropométricos em serviços de saúde: Norma Técnica do Sistema de Vigilância Alimentar e Nutricional - SISVAN. Brasília: Ministério da Saúde; 2011. 71 p.

13. World Health Organization. Obesity: preventing and managing the global epidemic. Geneva: WHO; 2000. 252 p. WHO Technical Report Series, n. 894.

14. Heyward VH, Stolarczyk LM. Avaliação da composição corporal aplicada. São Paulo: Manole; 2000. Capítulo 5.

15. Pedroso B, Pilatti LA, Gutierrez GL, Picinin CT. Cálculo dos escores e estatística descritiva do WHOQOL-bref através do Microsoft Excel. Rev Bras Qual Vida [Internet]. 2010; 2(01):31-6. [acesso em: 20 ago. 2015]. Disponível em: https://periodicos.utfpr.edu.br/rbqv/article/view/687/505

16. Brasil. Conselho Nacional de Saúde. Resolução 196/966. Aprova diretrizes e normas regulamentadoras de pesquisas envolvendo seres humanos. Diário Oficial da União 1996; 201(seção 1):21082. Disponível em: http://conselho.saude.gov.br/resolucoes/2012/Reso466.pdf

17. Liping M, Peng X, Haijiang L, Lahong J, Fan L. Quality of life of people Living with HIV/AIDS: A cross-sectional study in Zhejiang province, China. PLoS One 2015; 10(8): e0135705.

18. Valenti M, Masedu F, Mazza M, Tiberti S, Di Giovanni C, Calvarese A, et al. A longitudinal study of quality of life of earthquake survivors in L’Aquila, Italy. BMC Public Health 2013; 13(1143):1-7.

19. He Q, Yang L, Shi S, Gao J, Tao M, Zhang K, et al. Smoking and Major Depressive Disorder in Chinese Women. PLoS One 2014; 9(9):e106287.

20. Miranzi SSC, Ferreira FS, Iwamoto HH, Pereira GA, Miranzi MAS. Qualidade de vida em indivíduos com diabetes mellitus e hipertensão acompanhados por uma equipe de saúde da família. Texto Context Enferm. 2008; 17(4):672-29. 
21. Caspi A, Roberts BW, Shiner RL. Personality development: stability and change. Annu Rev Psychol. 2005; 56:453-84.

22. Roberts BW, Walton KE, Viechtbauer W. Patterns of mean-level change in personality traits across the life course: a meta-analysis of longitudinal studies. Psychol Bull. 2006; 132(1):1-25.

23. Marsh HW, Nagengast B, Morin AJS. Measurement invariance of Big-Five factors over the life span: ESEM tests of gender, age, plasticity, maturity, and La Dolce Vita effects. Dev Psychol. 2013; 49(6):1194-218.

24. Instituto Brasileiro de Geografia e Estatística. Pesquisa de Orçamentos Familiares 2008-2009. Antropometria e estado nutricional de crianças, adolescentes e adultos no Brasil [Internet]. Rio de Janeiro: IBGE; 2010 [acesso em: 14 ago. 2015]. Disponível em: http://www.ibge.gov.br/home/ estatistica/populacao/condicaodevida/pof/2008_2009_encaa/pof_20082009_encaa.pdf

25. Instituto Brasileiro de Geografia e Estatística. Coordenação de Trabalhos e Rendimentos. Pesquisa Nacional de Saúde 2013, Ciclos da Vida, Brasil e Grandes Regiões [Internet]. Rio de Janeiro: IBGE; 2015. 92 p. [acesso em: 22 set. 2015]. Disponível em: http://biblioteca.ibge.gov.br/visualizacao/ livros/liv94522.pdf

26. Jackson SE, Beeken RJ, Wardle J. Obesity, perceived weight discrimination, and psychological wellbeing in older adults in England. Obesity 2015; 23(5):1105-11.

27. Puhl RM, Heuer CA. Obesity stigma: important considerations for public health. Am J Public Health 2010; 100(6):1019-28.

28. Agrawal P, Gupta K, Mishra V, Agrawal S. The psychosocial factors related to obesity: a study among overweight, obese, and morbidly obese women in India. Women Health 2015; 55(44):623-45.

29. Cohen R, Blaszczynski A. Comparative effects of Facebook and conventional media on body image dissatisfaction. J Eat Disord. Journal of Eating Disorders; 2015; 3:23.

30. Chao H-L. Body image change in obese and overweight persons enrolled in weight loss intervention programs: A systematic review and meta-analysis. PLoS One 2015; 10(5):e0124036.

31. Jackson KL, Janssen I, Appelhans BM, Kazlauskaite R, Karavolos K, Dugan SA, et al. Body image satisfaction and depression in midlife women: the Study of Women's Health Across the Nation (SWAN). Arch Womens Ment Heal 2014; 17(3):117-87.

32. Runfola CD, Holle AV, Peat CM, Gagne DA, Brownley KA, Hofmeier SM, et al. Characteristics of women with body size satisfaction at midlife: results of the Gender and Body Image Study (GABI). J Women Aging 2013; 25(4):287-304.

33. Blake CE, Hébert JR, Lee DC, Adams SA, Steck SE, Sui X, et al. Adults with greater weight satisfaction report more positive health behaviors and have better health status regardless of BMI. J Obes 2013; 2013:13.

34. Prättälä R, Sippola R, Lahti-Koski M, Laaksonen MT, Mäkinen T, Roos E. Twenty-five year trends in body mass index by education and income in Finland. BMC Public Health 2012; 12(1):936. 
35. Mansfield ED, Ducharme N, Koski KG. Individual, social and environmental factors influencing physical activity levels and behaviours of multiethnic socio-economically disadvantaged urban mothers in Canada: a mixed methods approach. Int J Behav Nutr Phys Act 2012; 9(1):42.

36. Murtagh EM, Murphy MH, Murphy NM, Woods C, Nevill AM, Lane A. Prevalence and correlates of physical inactivity in community-dwelling older adults in Ireland. PLoS One 2015; 10(2):1-11.

37. Bryła M, Burzyńska M, Maniecka-Bryła I. Self-rated quality of life of city-dwelling elderly people benefitting from social help: results of a cross-sectional study. Health Qual Life Outcomes 2013;11:181.

38. Bourdieu P. Anamnese das constantes ocultas. In: Bourdieu P. A dominação masculina. 2 ed. Rio de Janeiro: Bertrand Brasil; 2002. Capítulo 2.

Recebido: 06/4/2016

Revisado: 18/9/2016

Aceito: 27/10/2016 
